\title{
Terapia física temprana en pacientes con lumbalgia de inicio reciente
}

Early physical therapy in adults with recent onset lumbar pain

\section{Objetivos}

Comparar la eficacia de la terapia física precoz versus la atención habitual en pacientes con dolor lumbar agudo atendidos en el ámbito de la atención primaria.

\section{Diseño y lugar}

Ensayo clínico aleatorizado y controlado. Fue llevado a cabo en Salt Lake City, Utah, EE. UU., entre 2011 y 2013.

\section{Pacientes}

Fueron seleccionados 220 pacientes con dolor lumbar de menos de 16 días de evolución que habían consultado a un profesional de atención primaria y que tenían buenas probabilidades de beneficiarse de un tratamiento de manipulación de acuerdo a los resultados de una regla de predicción clínica': 18 a 60 años de edad, dolor de menos de 16 días de evolución entre la duodécima costilla y los glúteos -con dolor o adormecimiento no más distales que la rodilla/s en las últimas 72 h-, y más de 20 puntos en el puntaje de Oswestry.

\section{Intervención}

Previamente a la asignación aleatoria, ambos grupos recibieron educación acerca del pronóstico favorable del dolor lumbar y consejos respecto de mantenerse lo más activos posible. Se les entregó una copia de "The Back Book", que contiene mensajes consistentes con las guías de manejo de dolor lumbar.

La asignación a los grupos fue a través de una lista de asi-
Fritz J y col. JAMA. 2015; 314(14):1459-1467. gnación aleatoria computarizada y fue ocultada a través de sobres sellados. Los dos grupos fueron:

a) Terapia física temprana $(n=108)$ : incluyó cuatro sesiones de evaluación y tratamiento. La primera incluyó manipulación de la columna (rotación y flexión) y ejercicios de movilidad de la misma, con prescripción de diez repeticiones, tres o cuatro veces al día para que el paciente realizara en su domicilio. La segunda incluyó manipulación, control de los ejercicios enseñados en la sesión anterior y prescripción de ejercicios de fortalecimiento de los músculos estabilizadores. Las últimas dos sesiones (tres y cuatro) fueron destinadas al control y a la progresión de los ejercicios prescriptos en las primeras dos.

b) Grupo control $(n=112)$ : no incluyó otra intervención más allá de la educación inicial.

\section{Medición de resultados principales}

La evaluación fue realizada antes de la intervención (basal o tiempo 0) y al mes, a los tres y a los 12 meses de instituida la misma.

El resultado primario fue el Puntaje de Discapacidad de Oswestry (0 a 100), en el que un mayor puntaje implica una mayor discapacidad y cuya diferencia mínima clínicamente relevante es de seis puntos.

El análisis fue realizado por intención de tratar.

\section{Resultados principales}

Los resultados principales se describen en la tabla 1.

Tabla 1: Resultados principales un ensayo clínico que evaluó la eficacia de la terapia física precoz en pacientes con lumbalgia de inicio reciente.

\begin{tabular}{|c|c|c|c|c|c|c|c|}
\hline \multirow[b]{2}{*}{ Resultado } & \multirow[b]{2}{*}{ Visita } & \multicolumn{2}{|c|}{ Control } & \multicolumn{2}{|c|}{ Terapia física precoz } & \multirow{2}{*}{$\begin{array}{c}\text { Diferencia } \\
\text { atribuible a la } \\
\text { terapia física } \\
\text { precoz }\end{array}$} & \multirow[b]{2}{*}{$\mathbf{p}$} \\
\hline & & $\begin{array}{l}\text { Puntaje } \\
\text { promedio }\end{array}$ & $\begin{array}{l}\text { Cambio promedio desde } \\
\text { el puntaje basal }\end{array}$ & $\begin{array}{l}\text { Puntaje } \\
\text { promedio }\end{array}$ & $\begin{array}{l}\text { Cambio promedio } \\
\text { desde el puntaje basal }\end{array}$ & & \\
\hline \multirow{4}{*}{$\begin{array}{l}\text { Puntaje de } \\
\text { discapacidad de } \\
\text { Oswestry } \\
\text { (IC95\%) }\end{array}$} & Basal & $40,9(38,6$ a 43,1) & & $41,3(38,7$ a 44,0$)$ & & & \\
\hline & Al mes & 14,5 (12,1 a 17,0) & $-26,6(-29,0$ a $-24,1)$ & $11,1(8,7 \mathrm{a} 13,4)$ & $-30,0(-32,4$ a $-27,7)$ & $-3,5(-6,8$ a $-0,08)$ & 0,045 \\
\hline & A los tres meses & $9,8$ (7,9a 11,7$)$ & $-31,3(-33,2 \mathrm{a}-29,4)$ & $6,6(4,7$ a 8,5$)$ & $-34,5(-36,4 a-32,6)$ & $-3,2(-5,9 a-0,47)$ & 0,02 \\
\hline & A los 12 meses & $9,8(6,8$ a 11,1$)$ & $-32,1(-34,3$ a $-30,0)$ & $7,0(4,8$ a 9,1$)$ & $-34,1(-36,3 a-32,0)$ & $-2,0(-5,0$ a 1,0$)$ & 0,19 \\
\hline
\end{tabular}

Puntaje de discapacidad de Oswestry: a mayor puntaje, peor funcionalidad (escala de 0 a 100).

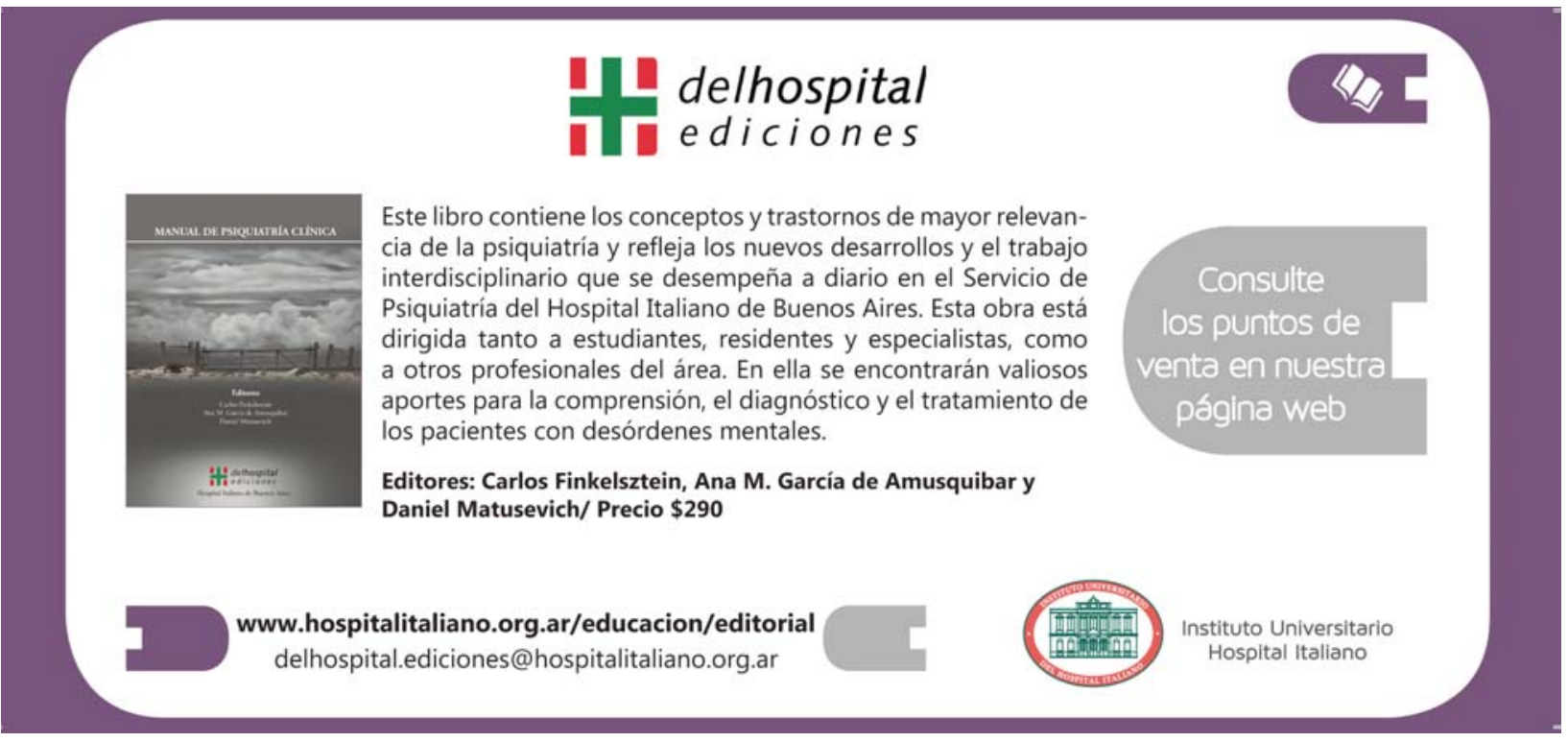




\section{Conclusiones}

La terapia física precoz resultó en una mejoría estadísticamente significativa en la discapacidad a los tres meses, pero la magnitud de la diferencia fue modesta y no alcanzó la mínima diferencia clínicamente importante. No hubo diferencias al año.
Fuente de Financiamiento: AHRQ; University of Utah Study Design and Biostatistics Center; National Center for Research Resources; National Center for Advancing Translational Sciences of the National Institutes of Health. Conflictos de Interés: Fritz reportó recibir honorarios de Focus On Therapeutic Outcomes.

\section{Comentario}

Si bien los autores de la investigación que hemos resumido procuraron evaluar la efectividad de dos tipos de intervenciones en pacientes con alta probabilidad de mejorar con terapia de manipulación -previamente identificados mediante una regla de predicción clínica ${ }^{2}$, no queda claro por qué definieron la rama "intervención" como una combinación de terapia manipulativa con ejercicios de movilidad y estabilización lumbar. Por otro lado, tampoco comunicaron el tipo de ejercicios realizados, la dirección de su movimiento, ni la progresión de fuerzas utilizadas. El 12\% de los individuos asignados al grupo intervención informaron efectos adversos atribuibles a la manipulación. Lamentablemente los autores no informan si hubo algún efecto adverso a los ejercicios prescriptos en cualquiera de las dos ramas ya que el grupo control también había recibido consejos sobre "mantenerse activo", en concordancia con las guías de práctica clínica. En este contexto, vale destacar que dichas guías de práctica clínica no establecen con precisión qué significa "mantenerse activo" ya que no toda actividad física es buena y algunas pueden ser incluso malas o funcionar como barreras hacia la recuperación ${ }^{3}$. Considero re- levante este punto ya que una mayor incidencia de efectos adversos en el grupo intervención podría haber sido responsable de diluir las diferencias de resultados entre ambos grupos.

\section{Conclusiones de la comentadora}

Dado que el dolor lumbar es un síntoma y no un diagnóstico, en los últimos años numerosos investigadores han establecido como prioridad identificar subgrupos de pacientes con dolor lumbar de diferentes características, con el objetivo de abandonar el uso de la etiqueta diagnóstica "dolor lumbar noespecífico", que suele englobar cerca del $85 \%$ de la población con dolor de espalda. Como documentaron Donelson y col. ${ }^{3}$, la identificación de diferentes subgrupos según la dirección de preferencia mecánica de posturas sostenidas y movimientos repetidos, es más segura y disminuye la probabilidad de dañar al paciente. Este esfuerzo por lograr una discriminación sindromática más fina es fundamental a la hora de comparar la efectividad de diferentes tratamientos ${ }^{3,4}$.

Carolina Guolo [ Servicio de Kinesiología del Hospital Italiano de Buenos Aires. carolina.guolo@ hiba.org.ar]

Guolo C. Terapia física temprana en pacientes con lumbalgia de inicio reciente. Evid Act Pract Ambul. 2016;19(2):45-46. Abr-Jun. Comentado de: Fritz J, col. Early physical therapy vs usual care in patients with recent-onset low back pain. JAMA. 2015; 314(14):1459-1467. PMID: 26461996.

\section{Referencias}

1.ChildsJ y col. A clinical prediction rule to identify patients with low back pain most likely to benefit from spinal manipulation: a validation study. Ann Intern Med. $2004 ; 141$ (12):920-928.

2. Flynn T y col. A clinical prediction rule for classifying patients with low back pain who demonstrate short-termimprovement with spinal manipulation, Spine (Phila Pa 1976), 2002; 27(24):2835-2843.

3. Donelson R y col. Rapidly Reversible Low Back Pain: An Evidence-Based Pathway to Widespread Recoveries and Savings, Hanover, NH: Self Care First; 2007.

4. Spratt K. Statistical relevance, In: Fardon DF, editor, Orthopaedic Knowledge Update: Spine, 2nd ed, Rosemont, IL: American Academy of Orthopaedic Surgeons; 2002.

\section{¡Vos también podes formar parte de esta transformación!}

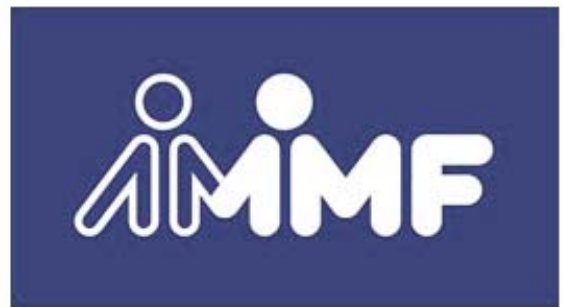

Asociación Metropolitana de Medicina Familiar.

Si queres asociarte o que te brindemos mayor información: secretaria.ammf@gmail.com

Seguinos en Facebook: www.facebook.com/AsociacionMetropolitanadeMedicinaFamiliar 